\title{
THE CONFORMAL YAMABE CONSTANT OF PRODUCT MANIFOLDS
}

\author{
BERND AMMANN, MATTIAS DAHL, AND EMMANUEL HUMBERT \\ (Communicated by Michael Wolf)
}

\begin{abstract}
Let $(V, g)$ and $(W, h)$ be compact Riemannian manifolds of dimension at least 3 . We derive a lower bound for the conformal Yamabe constant of the product manifold $(V \times W, g+h)$ in terms of the conformal Yamabe constants of $(V, g)$ and $(W, h)$.
\end{abstract}

\section{INTRODUCTION}

1.1. The Yamabe functional, constant scalar curvature metrics, and Yamabe metrics. For a Riemannian manifold $(M, G)$ we denote the scalar curvature by $s^{G}$, Laplace operator by $\Delta^{G}$, and volume form by $d v^{G}$. In general the dependence on the Riemannian metric is denoted by the metric as a superscript.

For integers $m \geq 3$ we set $a_{m}:=\frac{4(m-1)}{m-2}$ and $p_{m}:=\frac{2 m}{m-2}$. Let $C_{c}^{\infty}(M)$ denote the space of compactly supported smooth functions on $M$. For a Riemannian manifold $(M, G)$ of dimension $m \geq 3$ we define the Yamabe functional by

$$
\mathcal{F}^{G}(u):=\frac{\int_{M}\left(a_{m}|d u|_{G}^{2}+s^{G} u^{2}\right) d v^{G}}{\left(\int_{M}|u|^{p_{m}} d v^{G}\right)^{\frac{2}{p_{m}}}},
$$

where $u \in C_{c}^{\infty}(M)$ does not vanish identically. The conformal Yamabe constant $\mu(M, G)$ of $(M, G)$ is defined by

$$
\mu(M, G):=\inf _{u \in C_{c}^{\infty}(M), u \neq 0} \mathcal{F}^{G}(u) .
$$

The conformal Yamabe constant is usually defined only for compact manifolds; here we also allow non-compact manifolds in the definition. This will turn out to be essential for studying surgery formulas for Yamabe invariants of compact manifolds; see Subsection 3.2. Also notice that the conformal Yamabe constant for non-compact manifolds has been studied for instance in 13 and 11 .

For compact $M$ one easily sees that $\lim _{\varepsilon \rightarrow 0} \mathcal{F}^{G}\left(\sqrt{u^{2}+\varepsilon^{2}}\right)=\mathcal{F}^{G}(u)$; thus we obtain

$$
\mu(M, G)=\inf _{u \in C_{+}^{\infty}(M)} \mathcal{F}^{G}(u)>-\infty
$$

Received by the editors March 9, 2011 and, in revised form, June 22, 2011.

2010 Mathematics Subject Classification. Primary 35J60; Secondary 35P30, 58J50, 58C40.

Key words and phrases. Yamabe constant, Yamabe invariant, product manifolds.

The first author was partially supported by DFG Sachbeihilfe AM 144/2-1.

The second author was partially supported by the Swedish Research Council.

The third author was partially supported by ANR-10-BLAN 0105. 
where $C_{+}^{\infty}(M)$ denotes the space of positive smooth functions. According to the resolution of the Yamabe problem [23, 5, 21], see for example [14] for a good overview article, this infimum is always attained by a positive smooth function if $M$ is a compact manifold.

For a compact manifold $M$ one also defines for any metric $G$ the (normalized) Einstein-Hilbert functional $\mathcal{E}$ as

$$
\mathcal{E}(G):=\frac{\int_{M} s^{G} d v^{G}}{\operatorname{vol}^{G}(M)^{\frac{m-2}{m}}} .
$$

These functionals are closely related to each other; namely, if $u>0$ and $\widetilde{G}=$ $u^{4 /(m-2)} G$, then

$$
\mathcal{E}(\widetilde{G})=\mathcal{F}^{G}(u) .
$$

From the discussion above it follows that the functional $\mathcal{E}$ always attains its infimum in each conformal class $[G]$. Such minimizing metrics are called Yamabe metrics. Obviously $\widetilde{G}$ is a Yamabe metric if and only if $\lambda \widetilde{G}$ is a Yamabe metric for any $\lambda>0$. Thus any conformal class on a compact manifold carries a Yamabe metric of volume 1. Yamabe metrics $\widetilde{G}$ are stationary points of $\mathcal{E}$, restricted to the conformal class, and thus satisfy an Euler-Lagrange equation. This Euler-Lagrange equation says precisely that the scalar curvature of $\widetilde{G}$ is constant. One also sees that $\mu(M, G)$ is positive if and only if $[G]$ contains a metric of positive scalar curvature.

We denote the standard flat metric on $\mathbb{R}^{m}$ by $\xi^{m}$. The induced metric on the sphere $S^{m} \subset \mathbb{R}^{m+1}$ will be denoted by $\rho^{m}$. This metric is a Yamabe metric, and the whole orbit of $\rho^{m}$ under the action of the Möbius group $\operatorname{Conf}\left(S^{m}\right)=\operatorname{PSO}(m+1,1)$ consists of Yamabe metrics. Thus $\mathbb{S}^{m}:=\left(S^{m}, \rho^{m}\right)$ carries a non-compact space of Yamabe metrics of volume 1. It follows easily that $\mu\left(S^{m}, \rho^{m}\right)=m(m-1) \omega_{m}^{2 / m}$, where $\omega_{m}$ is the volume of $\left(S^{m}, \rho^{m}\right)$.

To determine the conformal Yamabe constant $\mu(M, G)$ it is obviously sufficient to know a Yamabe metric in $[G]$ explicitly. This is often easy to obtain in the following cases:

(i) If $M$ is compact and connected, and if $\mu(M, G) \leq 0$, then it follows from the maximum principle that there is a unique metric $g_{0} \in[G]$ of volume 1 and constant scalar curvature $s_{0}$. This then implies that $\mu(M, G)=s_{0}$.

(ii) Assume that $(M, G)$ is a connected compact Einstein manifold, and $(M, G)$ is non-isometric to $\left(S^{m}, \lambda \rho^{m}\right)$ for any $\lambda>0$. Then it was proven by Obata [15. Prop. 6.2] that $G$ contains a unique metric $g_{0}$ of volume 1 and constant scalar curvature $s_{0}$. Again $\mu(M, G)=s_{0}$.

(iii) If $(M, \tilde{G})$ is a metric of constant scalar curvature $\tilde{s}$ which is close in the $C^{2, \alpha}$-topology to an Einstein manifold $(M, G)$ as in (1.1), then it is proven by [9, Theorem $\mathrm{C}$ ] that $\tilde{G}$ is also a Yamabe metric, and thus $\mu(M, \tilde{G})=$ $\tilde{s} \operatorname{vol}(M, \tilde{G})^{2 / m}$. This applies for example to $(M, \tilde{G})=\left(S^{m}, \rho^{m}\right) \times\left(S^{m},(1+\right.$ $\left.\varepsilon) \rho^{m}\right), \varepsilon$ close to 0 . It also follows from the arguments in 9 that $\tilde{G}$ is then (up to rescaling) the only Yamabe metric in $[\tilde{G}]$. However, it is hard to decide in this situation whether $\tilde{G}$ is (up to rescaling) the only metric of constant scalar curvature in $[\tilde{G}]$. An affirmative answer to this problem was given recently in [10, Theorem 5] if at least one of the following additional conditions is satisfied: (a) $m \leq 7$; (b) $m \leq 24$ and $M$ is spin; or (c) $|W|+$ $|\nabla W|$ is a positive function. 
We have seen that in some particular cases, $\mu(M, g)$ can be explicitly calculated. In general the determination of $\mu(M, g)$ is a difficult task, as in most cases it is unclear whether a given constant scalar curvature metric in $[G]$ is a Yamabe metric. The functionals $\left.\mathcal{E}\right|_{[G]}$ and $\left.\mathcal{F}^{G}\right|_{C_{+}^{\infty}(M)}$ may have non-minimizing stationary points. These stationary points are thus metrics of constant scalar curvature which are not Yamabe metrics. The simplest such example, extensively discussed by Schoen 22 ] for $w=1$, is the metric $G=\rho^{v}+\lambda \rho^{w}$ on $S^{v} \times S^{w}, v \geq 2$, which has constant scalar curvature $v(v-1)+(1 / \lambda) w(w-1)$, but which is not a Yamabe metric for sufficiently large $\lambda$. This is due to the fact that $\mu(M, G) \leq \mu\left(\mathbb{S}^{m}\right)$, which follows from a standard test function argument, whereas $\mathcal{E}\left(\rho^{v}+\lambda \rho^{w}\right) \rightarrow \infty$ as $\lambda \rightarrow \infty$ when $v \geq 2$.

In conclusion, if $(M, G)$ is an explicitly given compact manifold of constant scalar curvature, then the calculation of $\mu(M, G)$ is easy if either $(M, G)$ is Einstein or if $\mu(M, G) \leq 0$, but in general it can be a hard problem.

1.2. Product manifolds. We now consider Riemannian product manifolds; that is, for Riemannian manifolds $(V, g)$ and $(W, h)$ of dimensions $v$ and $w$, we equip $M=V \times W$ with the product metric $G=g+h$, or more generally $G=g+\lambda h$, where $\lambda>0$. We ask the following question.

Question. Suppose $V$ and $W$ are compact and equipped with Yamabe metrics $g$ and $h$. Let $\lambda>0$. Is then $g+\lambda h$ also a Yamabe metric?

From the discussion on uniqueness above it follows that the answer is yes,

- if $v, w \geq 3, \mu(V, g) \leq 0$ and $\mu(W, h) \leq 0$;

- or if $v, w \geq 3, \mu(V, g)>0$ and $\mu(W, h)<0$ for $\lambda>0$ small enough;

- or if $(V, g)$ and $(W, h)$ are both Einstein with $\frac{1}{v} s^{g}$ close to $\frac{1}{\lambda w} s^{h}$.

If the answer to the above question is yes, then one deduces

$$
\mu(V \times W, g+\lambda h)=\left(\frac{\mu(V, g)}{\operatorname{vol}^{g}(V)^{2 / v}}+\frac{\mu(W, h)}{\operatorname{vol}^{\lambda h}(W)^{2 / w}}\right)\left(\operatorname{vol}^{g}(V) \operatorname{vol}^{\lambda h}(W)\right)^{\frac{2}{v+w}} .
$$

On the other hand if $g$ has positive scalar curvature, then $\mathcal{E}(g+\lambda h) \rightarrow \infty$ for $\lambda \rightarrow \infty$; thus $g+\lambda h$ is not a Yamabe metric for large $\lambda$. This applies, in particular, to the cases $\mu(V, g)>0, v \geq 3$, or if $(V, g)=\left(S^{2}, \rho^{2}\right)$.

1.3. An intuitive - but incorrect - argument in the positive case. Now we assume $v, w \geq 3, \mu(V, g)>0$, and $\mu(W, h)>0$. We already explained why $g+\lambda h$ is not a Yamabe metric for large (and small) $\lambda>0$, and as a consequence Equation (1) cannot be true for all $\lambda>0$. Despite this fact, assume for a moment that (1) were true for all $\lambda>0$. We then could minimize over $\lambda$, and we would obtain

$$
\inf _{\lambda \in(0, \infty)} \mu(V \times W, g+\lambda h)=(v+w)\left(\frac{\mu(V, g)}{v}\right)^{\frac{v}{v+w}}\left(\frac{\mu(W, h)}{w}\right)^{\frac{w}{v+w}} .
$$

1.4. Main result. Although the naive derivation of formula (2) used incorrect assumptions, our main result, Theorem 2.3. will tell us that the formula itself is correct up to a factor

$$
\varepsilon_{v, w}=\frac{a_{v+w}}{a_{v}^{v /(v+w)} a_{w} w /(v+w)}<1,
$$

assuming the mild condition (4). 
More precisely, we assume that $V$ and $W$ are compact manifolds of dimension at least 3, with Yamabe metrics $g$ and $h$ of positive conformal Yamabe constant. In particular, condition (4) is satisfied. Then Theorem 2.3 implies that

$$
\varepsilon_{v, w} \leq \frac{\inf _{\lambda \in(0, \infty)} \mu(V \times W, g+\lambda h)}{(v+w)\left(\frac{\mu(V, g)}{v}\right)^{\frac{v}{v+w}}\left(\frac{\mu(W, h)}{w}\right)^{\frac{w}{v+w}}} \leq 1 .
$$

Note that $\varepsilon_{v, w} \rightarrow 1$ for $v, w \rightarrow \infty$. See Figure 1 for some values of $\varepsilon_{v, w}$.

\begin{tabular}{r||l|l|l|l|l}
$\varepsilon_{v, w}$ & $w=3$ & $w=4$ & $w=5$ & $w=6$ & $w=7$ \\
\hline \hline$v=3$ & 0.625 & $0.7072 .$. & $0.7515 .$. & $0.7817 .$. & $0.8042 .$. \\
4 & $0.7072 .$. & $0.7777 .$. & $0.8007 .$. & $0.8367 .$. & $0.8537 .$. \\
5 & $0.7515 .$. & $0.8007 .$. & $0.8427 .$. & $0.8631 .$. & $0.8772 .$. \\
6 & $0.7817 .$. & $0.8367 .$. & $0.8631 .$. & 0.88 & $0.8921 .$. \\
7 & $0.8042 .$. & $0.8537 .$. & $0.8772 .$. & $0.8921 .$. & $0.9027 .$.
\end{tabular}

Figure 1. Values of $\varepsilon_{v, w}$

The main theorem also applies to many non-compact manifolds; see Theorem 2.3 .

1.5. Further comments on related literature. Our main motivation to study Yamabe constants of products is the application sketched in Subsection 3.2.

Fundamental results on Yamabe constants on products have been found in the interesting article [1, where it is, among other things, shown that the conformal Yamabe constant of the product $V \times \mathbb{R}^{w}$ is a lower bound for $\sigma(V \times W)$. Here $\sigma$ denotes the smooth Yamabe invariant defined in Subsection 3.1. This article also emphasized the importance of the question under which conditions a function $u \in C^{\infty}(V \times W)$ minimizing $\mathcal{F}$ is a function of only one of the factors. In [1] it is proved that if $(V, g)$ is compact and of constant scalar curvature 1 , then the infimum of the Yamabe functional of $V \times \mathbb{R}^{w}$ restricted to functions depending only on $\mathbb{R}^{w}$ is up to a constant the inverse of an optimal constant in a Gagliardo-Nirenberg type estimate.

In related research, Petean [17 derived a lower bound for the conformal Yamabe constant of product manifolds $V \times \mathbb{R}$, where $V$ is compact of positive Ricci curvature. If additionally we require $V$ to be Einstein, any minimizer $u \in C^{\infty}(V \times \mathbb{R})$ of $\mathcal{F}$ only depends on $\mathbb{R}$. As a corollary, Petean obtained lower bounds for the smooth Yamabe invariant $\sigma\left(V \times S^{1}\right)$ in this case.

This result of Petean contrasts nicely to Theorem 2.3. Whereas Petean's result requires that one of the factors is 1-dimensional, our Theorem 2.3 requires both factors to be of dimension at least 3 .

In [19] an explicit lower bound for $\mu\left(S^{2} \times \mathbb{R}^{2}, \rho^{2}+\xi^{2}\right)$ is obtained: $\mu\left(S^{2} \times \mathbb{R}^{2}, \rho^{2}+\right.$ $\left.\xi^{2}\right) \geq 0.68 \cdot Y\left(S^{4}\right)$. A similar but weaker result was obtained in [16.

Several recent publications study multiplicity phenomena on products $S^{v} \times W$ equipped with the product metric of the standard metric on $S^{v}$ with a metric of constant scalar curvature $s>0$ on $W$. Explicit lower bounds for the number of metrics of constant scalar curvature 1 in the conformal class $\left[g_{0}\right]$ are derived, and these bounds grow linearly in $\sqrt{s}$. The case $v=1$ was studied in [8, 7]; the general case then treated in [18]. In the recent preprint [12], isoparametric hypersurfaces are used in order to obtain new metrics of constant scalar curvature 
in the conformal class of products of Riemannian manifolds, e.g. the conformal class of $\left(S^{3} \times S^{3}, \rho^{3}+\lambda \rho^{3}\right)$.

1.6. Structure of the present article. In Section 2 we derive the main techniques and the main result of the article. We use mixed $L^{p, q}$-spaces in order to obtain a lower bound of the conformal Yamabe constants in the case that both factors have dimension at least 3 . We start with a proof of an iterated Hölder inequality in Subsection 2.1 which is well-adapted for the proof of our product formula in Subsection 2.3, which is the main result of the article.

In Section 3 we discuss applications. In Subsection 3.1 we find an estimate for the smooth Yamabe invariant of product manifolds. Subsection 3.2 explains our original motivation for the subject, which is to find better estimates for the constants appearing in the surgery formula in 2 . In Subsection 3.3 we define a stable Yamabe invariant and show that a similar surgery formula as in the unstable situation holds true.

\section{YAMABE CONSTANTS OF PRODUCT METRICS}

2.1. Iterated Hölder inequality for product manifolds. Let $(V, g)$ and $(W, h)$ be Riemannian manifolds of dimensions $v:=\operatorname{dim} V$ and $w:=\operatorname{dim} W$. We set

$$
(M, G):=(V \times W, g+h),
$$

so that $m:=\operatorname{dim} M=v+w$. We do not assume that the manifolds are complete. The first result we will need is a kind of iterated Hölder inequality for $(M, G):=$ $(V \times W, g+h)$.

Lemma 2.1. For any function $u \in C_{c}^{\infty}(M)$ we have

$$
\left(\int_{M}|u|^{p_{m}} d v^{G}\right)^{\frac{2}{p_{m}}} \leq\left(\int_{V}\left(\int_{W}|u|^{p_{w}} d v^{h}\right)^{\frac{2}{p_{w}}} d v^{g}\right)^{\frac{w}{m}}\left(\int_{V}\left(\int_{W}|u|^{2} d v^{h}\right)^{\frac{p_{v}}{2}} d v^{g}\right)^{\frac{v-2}{m}} .
$$

The lemma is actually a special case of the Hölder inequality for mixed $L^{p, q_{-}}$ spaces. See [6] for further information on such spaces.

Proof. By the Hölder inequality we have

$$
\int_{W}|u|^{p_{m}} d v^{h} \leq\left(\int_{W}|u|^{p_{w}} d v^{h}\right)^{\frac{w-2}{m-2}}\left(\int_{W}|u|^{2} d v^{h}\right)^{\frac{v}{m-2}} .
$$

We integrate this inequality over $(V, g)$ and use the following Hölder inequality:

$$
\int_{V} \alpha \beta d v^{g} \leq\left(\int_{V}|\alpha|^{\frac{m-2}{w}} d v^{g}\right)^{\frac{w}{m-2}}\left(\int_{V}|\beta|^{\frac{m-2}{v-2}} d v^{g}\right)^{\frac{v-2}{m-2}}
$$

with

$$
\alpha:=\left(\int_{W}|u|^{p_{w}} d v^{h}\right)^{\frac{w-2}{m-2}} \text { and } \beta:=\left(\int_{W}|u|^{2} d v^{h}\right)^{\frac{v}{m-2}} .
$$

This proves Lemma 2.1 . 
2.2. A lemma about integration and derivation. Second we need a lemma concerning the interchange of derivation and taking (a partial) $L^{2}$-norm.

Lemma 2.2. Let $u \in C_{c}^{\infty}(M), u \not \equiv 0$, and set

$$
\gamma:=\left(\int_{W} u^{2} d v^{h}\right)^{\frac{1}{2}} .
$$

Then

$$
\int_{V}|d \gamma|_{g}^{2} d v^{g} \leq \int_{M}|d u|_{g}^{2} d v^{G}
$$

Proof. Take any vector field $X$ on $M$ tangent to $V$. One has $g$-almost everywhere (except on the boundary of $\gamma^{-1}(0)$ )

$$
|X \gamma|^{2} \leq\left(\frac{\int_{W} u X u d v^{h}}{\left(\int_{W} u^{2} d v^{h}\right)^{\frac{1}{2}}}\right)^{2} \leq \int_{W}(X u)^{2} d v^{h},
$$

where we used the Cauchy-Schwartz inequality

$$
\int_{W} u X u d v^{h} \leq\left(\int_{W}(X u)^{2} d v^{h}\right)^{\frac{1}{2}}\left(\int_{W} u^{2} d v^{h}\right)^{\frac{1}{2}} .
$$

Integrating over $V$, we deduce that

$$
\int_{V}|X \gamma|^{2} d v^{g} \leq \int_{M}|X u|^{2} d v^{G}
$$

Since this holds for any $X$ tangent to $V$, inequality (3) follows.

2.3. Conformal Yamabe constant of product metrics. We now state and prove our main theorem. It will turn out that the following modified invariant is convenient when studying products of Riemannian manifolds with non-negative Yamabe constant. If $\mu(M, G) \geq 0$ we set

$$
\nu(M, G):=\left(\frac{\mu(M, G)}{m a_{m}}\right)^{m} .
$$

Theorem 2.3. Let $(V, g)$ and $(W, h)$ be Riemannian manifolds of dimensions $v, w \geq 3$. Assume that $\mu(V, g), \mu(W, h) \geq 0$ and that

$$
\frac{s^{g}+s^{h}}{a_{m}} \geq \frac{s^{g}}{a_{v}}+\frac{s^{h}}{a_{w}} .
$$

Then,

or, equivalently,

$$
\mu(M, G) \geq \frac{m a_{m}}{\left(v a_{v}\right)^{\frac{v}{m}}\left(w a_{w}\right)^{\frac{w}{m}}} \mu(V, g)^{\frac{v}{m}} \mu(W, h)^{\frac{w}{m}},
$$

$$
\nu(M, G) \geq \nu(V, g) \nu(W, h) .
$$

Note that we do not assume that the manifolds are complete.

Proof. Take any non-negative function $u \in C_{c}^{\infty}(M)$ normalized by

$$
\int_{M}|u|^{p_{m}} d v^{G}=1
$$


We then have

$$
\frac{1}{a_{m}} \mathcal{F}^{G}(u)=\int_{M}\left(|d u|_{G}^{2}+\frac{s^{G}}{a_{m}} u^{2}\right) d v^{G} .
$$

Using $|d u|_{G}^{2}=|d u|_{g}^{2}+|d u|_{h}^{2}$ and $s^{G}=s^{g}+s^{h}$ together with (4) we obtain

$$
\frac{1}{a_{m}} \mathcal{F}^{G}(u) \geq \int_{M}\left(|d u|_{g}^{2}+\frac{s^{g}}{a_{v}} u^{2}\right) d v^{G}+\int_{V} \int_{W}\left(|d u|_{h}^{2}+\frac{s^{h}}{a_{w}} u^{2}\right) d v^{h} d v^{g} .
$$

We set $\gamma:=\left(\int_{W} u^{2} d v^{h}\right)^{\frac{1}{2}}$. For the first term here, Lemma 2.2 and the definition of $\mu(V, g)$ imply that

$$
\begin{aligned}
\int_{M}\left(|d u|_{g}^{2}+\frac{s^{g}}{a_{v}} u^{2}\right) d v^{G} & \geq \int_{V}\left(|d \gamma|_{g}^{2}+\frac{s^{g}}{a_{v}} \gamma^{2}\right) d v^{g} \\
& \geq \frac{1}{a_{v}} \mu(V, g)\left(\int_{V} \gamma^{p_{v}} d v^{g}\right)^{\frac{2}{p_{v}}} \\
& =\frac{1}{a_{v}} \mu(V, g)\left(\int_{V}\left(\int_{W}|u|^{2} d v^{h}\right)^{\frac{p_{v}}{2}} d v^{g}\right)^{\frac{v-2}{v}} .
\end{aligned}
$$

For the second term we have

$$
\int_{V} \int_{W}\left(|d u|_{h}^{2}+\frac{s^{h}}{a_{w}} u^{2}\right) d v^{h} d v^{g} \geq \frac{1}{a_{w}} \mu(W, h) \int_{V}\left(\int_{W} u^{p_{w}} d v^{h}\right)^{\frac{2}{p_{w}}} d v^{g}
$$

by the definition of $\mu(W, h)$. Plugging (7) and (8) into (6) we get

$$
\begin{aligned}
\mathcal{F}^{G}(u) \geq \frac{a_{m}}{a_{v}} \mu(V, g)\left(\int_{V}\left(\int_{W}|u|^{2} d v^{h}\right)^{\frac{p_{v}}{2}} d v^{g}\right)^{\frac{v-2}{v}} \\
+\frac{a_{m}}{a_{w}} \mu(W, h) \int_{V}\left(\int_{W} u^{p_{w}} d v^{h}\right)^{\frac{2}{p_{w}}} d v^{g}
\end{aligned}
$$

Set

$$
r:=m a_{m} \nu(V, g)^{\frac{1}{m}} \nu(W, h)^{\frac{1}{m}} .
$$

For $a, b>0$ we compute

$$
\begin{aligned}
r a^{\frac{v-2}{m}} b^{\frac{w}{m}}= & r\left(\left(\frac{\nu(V, g)^{w}}{\nu(W, h)^{v}}\right)^{\frac{1}{m^{2}}} a^{\frac{v-2}{m}}\right)\left(\left(\frac{\nu(W, h)^{v}}{\nu(V, g)^{w}}\right)^{\frac{1}{m^{2}}} b^{\frac{w}{m}}\right) \\
\leq & r\left[\frac{v}{m}\left(\frac{\nu(V, g)^{\frac{w}{v}}}{\nu(W, h)}\right)^{\frac{1}{m}} a^{\frac{v-2}{v}}+\frac{w}{m}\left(\frac{\nu(W, h)^{\frac{v}{w}}}{\nu(V, g)}\right)^{\frac{1}{m}} b\right] \\
= & m a_{m} \nu(V, g)^{\frac{1}{m}} \nu(W, h)^{\frac{1}{m}} \frac{v}{m}\left(\frac{\nu(V, g)^{\frac{w}{v}}}{\nu(W, h)}\right)^{\frac{1}{m}} a^{\frac{v-2}{v}} \\
& \quad+m a_{m} \nu(V, g)^{\frac{1}{m}} \nu(W, h)^{\frac{1}{m}} \frac{w}{m}\left(\frac{\nu(W, h)^{\frac{v}{w}}}{\nu(V, g)}\right)^{\frac{1}{m}} b \\
= & a_{m} v \nu(V, g)^{\frac{1}{v}} a^{\frac{v-2}{v}}+a_{m} w \nu(W, h)^{\frac{1}{w}} b \\
= & \frac{a_{m}}{a_{v}} \mu(V, g) a^{\frac{v-2}{v}}+\frac{a_{m}}{a_{w}} \mu(W, h) b,
\end{aligned}
$$


where in the second line we used Young's inequality

$$
c d \leq \frac{v}{m} c^{\frac{m}{v}}+\frac{w}{m} d^{\frac{m}{w}},
$$

which is valid for any $c, d \geq 0$. Using the above in (9) with

$$
a:=\int_{V}\left(\int_{W}|u|^{2} d v^{h}\right)^{\frac{p_{v}}{2}} d v^{g} \text { and } b:=\int_{V}\left(\int_{W}|u|^{p_{w}} d v^{h}\right)^{\frac{2}{p_{w}}} d v^{g},
$$

we get

$$
\mathcal{F}^{G}(u) \geq r\left(\int_{V}\left(\int_{W}|u|^{2} d v^{h}\right)^{\frac{p_{v}}{2}} d v^{g}\right)^{\frac{v-2}{m}}\left(\int_{V}\left(\int_{W}|u|^{p_{w}} d v^{h}\right)^{\frac{2}{p_{w}}} d v^{g}\right)^{\frac{w}{m}} .
$$

Using Lemma 2.1 and Relation (5) we deduce

$$
\mathcal{F}^{G}(u) \geq r=m a_{m} \nu(V, g)^{\frac{1}{m}} \nu(W, h)^{\frac{1}{m}}=\frac{m a_{m}}{\left(v a_{v}\right)^{\frac{v}{m}}\left(w a_{w}\right)^{\frac{w}{m}}} \mu(V, g)^{\frac{v}{m}} \mu(W, h)^{\frac{w}{m}} .
$$

Since this holds for all $u$, Theorem 2.3 follows.

\section{Applications}

3.1. The smooth Yamabe invariant of product manifolds. Let $M$ be a compact manifold of dimension $m \geq 3$. Then its smooth Yamabe invariant is defined as

$$
\sigma(M):=\sup \mu(M, G),
$$

where the supremum runs over all Riemannian metrics $G$ on $M$. This invariant of differentiable manifolds has the property that $\sigma(M) \leq \sigma\left(S^{m}\right)$ for all $M$ and $\sigma(M)>0$ if and only if $M$ admits a metric with positive scalar curvature.

From Theorem 2.3 we obtain the following corollary.

Corollary 3.1. Let $V, W$ be compact manifolds of dimensions $v, w \geq 3$. Assume $\sigma(V) \geq 0$. Then

$$
\sigma(V \times W) \geq \frac{m a_{m}}{\left(v a_{v}\right)^{\frac{v}{m}}\left(w a_{w}\right)^{\frac{w}{m}}} \sigma(V)^{\frac{v}{m}} \sigma\left(S^{w}\right)^{\frac{w}{m}},
$$

where $m=v+w$.

Proof. We first consider the case $\sigma(V)>0$. In [1, Theorem 1.1] it is proven that

$$
\lim _{t \rightarrow \infty} \mu\left(V \times W, g+t^{2} h\right)=\mu\left(V \times \mathbb{R}^{w}, g+\xi^{w}\right)
$$

if $g$ is a metric on $V$ with positive scalar curvature and $h$ is any metric on $W$. Since $a_{v} \geq a_{m}$ we see that (4) holds, so Theorem 2.3 together with $\mu\left(\mathbb{R}^{w}, \xi^{w}\right)=\mu\left(S^{w}, \rho^{w}\right)$ implies the corollary if $\sigma(V)>0$.

In the case $\sigma(V)=0$ there is a sequence of metrics $g_{i}$ on $V$ such that vol ${ }^{g_{i}}(V)=1$, $\mu\left(V, g_{i}\right) \leq 0$, and $\mu\left(V, g_{i}\right) \rightarrow 0$ as $i \rightarrow \infty$. From the solution of the Yamabe problem we can assume that all $g_{i}$ have constant scalar curvature $s^{g_{i}}=\mu\left(V, g_{i}\right)$. Choose $\varepsilon_{i}>0$ such that $\varepsilon_{i} \rightarrow 0$ and $\varepsilon_{i}^{-w} \mu\left(V, g_{i}\right) \rightarrow 0$ for $i \rightarrow \infty$. For a unit volume metric $h$ on $W$ with constant scalar curvature $s^{h}$, the metric $G_{i}:=\varepsilon_{i}^{w} g_{i}+\varepsilon_{i}^{-v} h$ has $\operatorname{vol}^{G_{i}}(V \times W)=1$ and constant scalar curvature $\varepsilon_{i}^{-w} \mu\left(V, g_{i}\right)+\varepsilon_{i}^{v} s^{h} \rightarrow 0$. It follows that $\mu\left(V \times W, G_{i}\right) \rightarrow 0$ and thus $\sigma(V \times W) \geq 0$. 
3.2. Surgery formulas. Assume that $M$ is a compact $m$-dimensional manifold and that $i: S^{k} \times \overline{B^{m-k}} \rightarrow M$ is an embedding. We define

$$
N:=\left(M \backslash i\left(S^{k} \times B^{m-k}\right) \cup_{\partial}\left(B^{k+1} \times S^{n-k-1}\right),\right.
$$

where $\cup_{\partial}$ means that we identify $x \in S^{k} \times S^{m-k-1}=\partial\left(B^{k+1} \times S^{m-k-1}\right)$ with $i(x) \in \partial i\left(S^{k} \times B^{m-k}\right)$. After a smoothing procedure, $N$ is again a compact manifold without boundary, and we say that $N$ is obtained from $M$ by m-dimensional surgery along $i$.

In [2, Corollary 1.4] we found the following result.

Theorem 3.2. Let $N$ be obtained from $M$ via surgery of dimension $k \in\{0,1, \ldots$, $m-3\}$. Then there is a constant $\Lambda_{m, k}>0$ with

$$
\sigma(N) \geq \min \left\{\sigma(M), \Lambda_{m, k}\right\} .
$$

Furthermore, for $k=0$ this statement is true for $\Lambda_{m, 0}=\infty$.

It is helpful to consider how the constant $\Lambda_{m, k}$ was obtained in [2] in the case $k \geq 1$. We showed that Theorem 3.2 holds for a constant $\Lambda_{m, k}$ satisfying

$$
\Lambda_{m, k} \geq \min \left\{\Lambda_{m, k}^{(1)}, \Lambda_{m, k}^{(2)}\right\}
$$

We will not recall here the definitions of $\Lambda_{m, k}^{(1)}$ and $\Lambda_{m, k}^{(2)}$ in detail, as they are not needed, but we will explain some relevant facts for $\Lambda_{m, k}^{(1)}$ and $\Lambda_{m, k}^{(2)}$.

For $c \in[0,1]$, let $\mathbb{H}_{c}^{k+1}$ be the simply connected $(k+1)$-dimensional complete Riemannian manifold of constant sectional curvature $-c^{2}$; for $c=0$ it is $\mathbb{R}^{k+1}$ and for $c>0$ it is hyperbolic space rescaled by a factor $c^{-2}$. One defines

$$
\Lambda_{m, k}^{(0)}:=\inf _{c \in[0,1]} \mu\left(\mathbb{H}_{c}^{k+1} \times \mathbb{S}^{m-k-1}\right) .
$$

It was shown in [2, Corollary 1.4] that $\Lambda_{m, k}^{(1)} \geq \Lambda_{m, k}^{(0)}$ for $k \in\{1, \ldots, m-3\}$. Furthermore $\Lambda_{m, k}^{(2)} \geq \Lambda_{m, k}^{(1)}$ will be shown in our publication [4] provided that $k+3 \leq$ $m \leq 5$ or $k+4 \leq m$. Thus Theorem 3.2 holds for $\Lambda_{m, k}:=\Lambda_{m, k}^{(0)}$ if $k+3 \leq m \leq 5$ or $k+4 \leq m$.

Since the manifolds $\mathbb{H}_{c}^{k+1} \times \mathbb{S}^{m-k-1}$ with $c^{2} \leq 1$ satisfy Condition (41) and since $\mu\left(\mathbb{H}_{c}^{k+1}\right)=\mu\left(\mathbb{S}^{k+1}\right)$, we obtain the following corollary from Theorem 2.3 ,

Corollary 3.3. If $2 \leq k \leq m-4$, then Theorem 3.2 holds for

$$
\Lambda_{m, k}=\frac{m a_{m}}{\left((k+1) a_{k+1}\right)^{\frac{k+1}{m}}\left((m-k-1) a_{m-k-1}\right)^{\frac{m-k-1}{m}}} \sigma\left(S^{k+1}\right)^{\frac{k+1}{m}} \sigma\left(S^{m-k-1}\right)^{\frac{m-k-1}{m}} .
$$

It follows for example: If $M$ is an $m$-dimensional compact manifold, obtained from $S^{m}$ by performing successive surgeries of dimension $k, 0 \leq k \leq m-4, k \neq 1$, then $\sigma(M) \geq \Lambda_{m}$, where $\Lambda_{6}=54.779, \Lambda_{7}=74.504, \Lambda_{8}=92.242, \Lambda_{9}=109.426$, etc.

Another technique presented in [3] will allow us to control the effect of 1dimensional surgeries. In combination with [19] and [20] one obtains $\Lambda_{4,1}>38.9$ and $\Lambda_{5,1}>45.1$. 
3.3. A stable Yamabe invariant. In this section we will define and discuss a "stabilized" Yamabe invariant, obtained by letting the dimension go to infinity for a given compact Riemannian manifold by multiplying with Ricci-flat manifolds of increasing dimension. Very optimistically, such a stabilization could be related to the linear eigenvalue problem obtained by formally letting the dimension tend to infinity in the Yamabe problem. The stable invariant can also be viewed as a quantitative refinement of the property that a given manifold admit stably positive scalar curvature.

For a compact manifold $M$ with $\sigma(M) \geq 0$ we define

$$
\Sigma(M):=\left(\frac{\sigma(M)}{m a_{m}}\right)^{m} .
$$

Then

$$
\Sigma(M)=\sup \nu(M, G),
$$

where the supremum runs over all Riemannian metrics $G$ on $M$. The conclusion of Corollary 3.1 can be formulated as

$$
\Sigma(V \times W) \geq \Sigma(V) \Sigma\left(S^{w}\right) .
$$

Let $(B, \beta)$ be a compact Ricci-flat manifold of dimension $b$. We could for example choose $B$ to be the 1-dimensional circle $S^{1}$, or an 8 -dimensional Bott manifold equipped with a metric with holonomy $\operatorname{Spin}(7)$. From (10) we then get

$$
\frac{\Sigma\left(S^{v+b i}\right)}{\Sigma\left(S^{b i}\right)} \geq \frac{\Sigma\left(V \times B^{i}\right)}{\Sigma\left(S^{b i}\right)} \geq \Sigma(V)
$$

where the upper bound comes from $\Sigma\left(V \times B^{i}\right) \leq \Sigma\left(S^{v+b i}\right)$. We define the stable Yamabe invariant of $V$ as the limit superior of the middle term,

$$
\bar{\Sigma}(V):=\limsup _{i \rightarrow \infty} \frac{\Sigma\left(V \times B^{i}\right)}{\Sigma\left(S^{b i}\right)} .
$$

To see that the stable Yamabe invariant is finite we need to study the upper bound in (11) and the function $v \mapsto \Sigma\left(S^{v}\right)$. We have

$$
\sigma\left(S^{v}\right)=v(v-1) \omega_{v}^{2 / v}, \quad \omega_{v}=\frac{2 \pi^{\frac{v+1}{2}}}{\Gamma\left(\frac{v+1}{2}\right)},
$$

where $\omega_{v}$ is the volume of $\mathbb{S}^{v}$, so

$$
\Sigma\left(S^{v}\right)=4 \pi\left(\frac{\pi(v-2)}{4}\right)^{v} \frac{1}{\Gamma\left(\frac{v+1}{2}\right)^{2}} .
$$

Stirling's formula tells us that

$$
\Gamma(z)=\sqrt{\frac{2 \pi}{z}}\left(\frac{z}{e}\right)^{z}\left(1+O\left(\frac{1}{z}\right)\right)
$$

and therefore

$$
\begin{aligned}
\Sigma\left(S^{v}\right) & =4 \pi\left(\frac{\pi(v-2)}{4}\right)^{v} \frac{v+1}{4 \pi}\left(\frac{2 e}{v+1}\right)^{v+1}\left(1+O\left(\frac{1}{v}\right)\right) \\
& =2 e\left(\frac{\pi e}{2}\right)^{v} \frac{(1-2 / v)^{v}}{(1+1 / v)^{v}}\left(1+O\left(\frac{1}{v}\right)\right) \\
& =2 e^{-2}\left(\frac{\pi e}{2}\right)^{v}\left(1+O\left(\frac{1}{v}\right)\right) .
\end{aligned}
$$


We see that

$$
\lim _{i \rightarrow \infty} \frac{\Sigma\left(S^{v+b i}\right)}{\Sigma\left(S^{b i}\right)}=\lim _{i \rightarrow \infty}\left(\frac{\pi e}{2}\right)^{v}\left(1+O\left(\frac{1}{b i}\right)\right)=\left(\frac{\pi e}{2}\right)^{v}
$$

so from (11) we get the following bound on the stable Yamabe invariant:

$$
\left(\frac{\pi e}{2}\right)^{v} \geq \bar{\Sigma}(V) \geq \Sigma(V)
$$

We conclude that the stable invariant is a non-trivial invariant.

The stable Yamabe invariant is not strictly speaking a stable invariant in the sense that it gives the same value for $V$ and $V \times B^{i}$. These values are however related by a simple identity, as we will see next. Taking the limit superior as $j \rightarrow \infty$ in

$$
\frac{\Sigma\left(V \times B^{i} \times B^{j}\right)}{\Sigma\left(S^{b j}\right)}=\frac{\Sigma\left(V \times B^{i+j}\right)}{\Sigma\left(S^{b i+b j}\right)} \frac{\Sigma\left(S^{b i+b j}\right)}{\Sigma\left(S^{b j}\right)},
$$

we conclude that

$$
\bar{\Sigma}\left(V \times B^{i}\right)=\bar{\Sigma}(V)\left(\frac{\pi e}{2}\right)^{b i}
$$

and further

$$
\bar{\Sigma}(V) \geq \Sigma\left(V \times B^{i}\right)\left(\frac{\pi e}{2}\right)^{-b i}
$$

for all $i \geq 0$.

The next simple proposition tells us that the positivity of $\Sigma(V)$ is equivalent to $V$ having stable metrics of positive scalar curvature.

Proposition 3.4. Let $V$ be a compact manifold. The following three statements are equivalent.

(a) $\bar{\Sigma}(V)>0$.

(b) There is $i_{0}>0$ such that $V \times B^{i_{0}}$ admits a positive scalar curvature metric.

(c) There is a $i_{0}>0$ such that $V \times B^{i}$ admits a positive scalar curvature metric for all $i \geq i_{0}$.

Proof. The implications $(a) \Rightarrow(b)$ and $(b) \Leftrightarrow(c)$ are easy to show. The implication $(b) \Rightarrow(a)$ is a consequence of (12).

We also obtain a stable version of Theorem 3.2 for surgeries of codimension at least 4. A similar result holds for surgeries of codimension 3, but with a less explicit constant.

Theorem 3.5. Assume that $N$ is obtained from the compact m-dimensional manifold $M$ by surgery of dimension $k$, where $0 \leq k \leq m-4$. Then

$$
\bar{\Sigma}(N) \geq \min \left\{\bar{\Sigma}(M), \bar{\Sigma}\left(S^{m}\right),\left(\frac{\pi e}{2}\right)^{k+1} \Sigma\left(S^{m-k-1}\right)\right\} .
$$

Proof. The manifold $N$ after surgery is obtained by a connected sum of $M$ and $S^{m}$ along embeddings of a $k$-dimensional sphere with trivial normal bundle. Similarly $N \times B^{i}$ is obtained by a connected sum of $M \times B^{i}$ and $S^{m} \times B^{i}$ by a connected sum 
along embeddings of $S^{k} \times B^{i}$ with trivial normal bundle. Thus [2, Theorem 1.3] together with Corollary 3.3 tells us that

$$
\begin{aligned}
\Sigma\left(N \times B^{i}\right) & \geq \min \left\{\Sigma\left(M \times B^{i}\right), \Sigma\left(S^{m} \times B^{i}\right),\left(\frac{\Lambda_{m+b i, k+b i}}{(m+b i) a_{m+b i}}\right)^{m+b i}\right\} \\
& \geq \min \left\{\Sigma\left(M \times B^{i}\right), \Sigma\left(S^{m} \times B^{i}\right), \Sigma\left(S^{k+b i+1}\right) \Sigma\left(S^{m-k-1}\right)\right\}
\end{aligned}
$$

and this yields the statement of the theorem.

For the smooth Yamabe invariant the value of the sphere is a universal upper bound. One can ask if the same holds for the stable invariant: is $\bar{\Sigma}(M) \leq \bar{\Sigma}\left(S^{m}\right)$ for all $M$ ?

\section{ACKNOWLEDGEMENTS}

We want to thank the organizers of the conference "Contributions in Differential Geometry - a round table on the occasion of the 65th birthday of Lionel Bérard Bergery, 2010", in particular thanks to A. Besse, A. Altomani, T. Krantz and M.-A. Lawn. During that conference we found central ingredients for the present article. We thank P. Piccione for pointing out an error in a preprint version of this article. Further, we would like to thank the anonymous referee for many helpful comments.

\section{REFERENCES}

1. K. Akutagawa, L. Florit, and J. Petean, On the Yamabe constant of Riemannian products, Comm. Anal. Geom. 15 (2007), 947-969. MR2403191 (2009i:53030)

2. B. Ammann, M. Dahl, and E. Humbert, Smooth Yamabe invariant and surgery, Preprint, arXiv 0804.1418, 2008.

3. L_Lw-dimensional surgery and the Yamabe invariant, Preprint in preparation, 2011.

4. _ Square-integrability of solutions of the Yamabe equation, Preprint, arXiv:1111.2780, 2011.

5. T. Aubin, Équations différentielles non linéaires et problème de Yamabe concernant la courbure scalaire, J. Math. Pure Appl., IX. Ser. 55 (1976), 269-296. MR0431287 (55:4288)

6. A. Benedek and R. Panzone, The space $L^{p}$, with mixed norm, Duke Math. J. 28 (1961), 301-324. MR0126155 (23:A3451)

7. L. Bérard Bergery and G. Kaas, Examples of multiple solutions for the Yamabe problem on scalar curvature, Preprint, http://hal.archives-ouvertes.fr/hal-00143495/.

8. _ Remark on an example by $R$. Schoen concerning the scalar curvature, Preprint, http://hal.archives-ouvertes.fr/hal-00143485/.

9. C. Böhm, M. Wang, and W. Ziller, A variational approach for compact homogeneous Einstein manifolds, Geom. Funct. Anal. 14 (2004), 681-733. MR2084976 (2005g:53074)

10. L. L. de Lima, P. Piccione, and M. Zedda, A note on the uniqueness of solutions for the Yamabe problem, to appear in Proc. Amer. Math. Soc., 2011, arXiv:1102.2321.

11. N. Große, The Yamabe equation on manifolds of bounded geometry, Preprint, 2009, arXiv:0912.4398.

12. G. Henry and J. Petean, Isoparametric hypersurfaces and metrics of constant scalar curvature, Preprint, CIMAT Mexico, 2011.

13. S. Kim, An obstruction to the conformal compactification of Riemannian manifolds, Proc. Amer. Math. Soc. 128 (2000), no. 6, 1833-1838. MR1646195 (2000j:53048)

14. J. M. Lee and T. H. Parker, The Yamabe problem, Bull. Amer. Math. Soc., New Ser. 17 (1987), 37-91. MR888880 (88f:53001)

15. M. Obata, The conjectures on conformal transformations of Riemannian manifolds, J. Diff. Geom. 6 (1971/72), 247-258. MR0303464 (46:2601)

16. J. Petean, Best Sobolev constants and manifolds with positive scalar curvature metrics, Ann. Global Anal. Geom. 20 (2001), 231-242. MR.1866416 (2002h:53064) 
17. Isoperimetric regions in spherical cones and Yamabe constants of $M \times S^{1}$, Geom. Dedicata 143 (2009), 37-48. MR2576291 (2011b:53078)

18. Metrics of constant scalar curvature conformal to Riemannian products, Proc. Amer. Math. Soc. 138 (2010), 2897-2905. MR2644902

19. J. Petean and J. M. Ruiz, Isoperimetric profile comparisons and Yamabe constants, Ann. Global Anal. Geom. 40 (2011), 177-189. MR2811624

20. - On the Yamabe constants of $S^{2} \times R^{3}$ and $S^{3} \times R^{2}$, Preprint, arXiv:1202.1022, 2011.

21. R. Schoen, Conformal deformation of a Riemannian metric to constant scalar curvature, J. Diff. Geom. 20 (1984), 479-495. MR788292 (86i:58137)

22. On the number of constant scalar curvature metrics in a conformal class, Differential geometry, Pitman Monogr. Surveys Pure Appl. Math., vol. 52, Longman Sci. Tech., Harlow, 1991, pp. 311-320. MR.1173050 (94e:53035)

23. N. S. Trudinger, Remarks concerning the conformal deformation of Riemannian structures on compact manifolds, Ann. Sc. Norm. Super. Pisa, Sci. Fis. Mat., III. Ser. 22 (1968), 265-274. MR.0240748(39:2093)

FAkUltät Für Mathematik, Universität Regensburg, 93040 Regensburg, Germany

E-mail address: bernd.ammann@mathematik.uni-regensburg.de

Institutionen för Matematik, Kungliga Tekniska Högskolan, 10044 Stockholm, Sweden

E-mail address: dahl@math.kth.se

Institut Élie Cartan, BP 239, Université de Nancy 1, 54506 Vandoeuvre-Lès-Nancy Cedex, France

Current address: Laboratoire de Mathématiques et Physique Théorique, Université de Tours, Parc de Grandmot, 37200 Tours, France

E-mail address: emmanuel.humbert@lmpt.univ-tours.fr 